\title{
利用双流体模型研究柴油高压喷嘴内部的空化流动
}

\author{
汪翔，苏万华
}

天津大学内燃机燃烧学国家重点实验室, 天津 300072

E-mail: waylandwx@yahoo.com.cn

2007-10-23 收稿, 2008-07-10 接受

国家自然科学基金重点项目(编号: 50636040)和国家重点基础研究发展计划(编号: 2007CB210001)资助

摘要 利用双流体模型对一单孔柴油喷嘴内部复杂的空化流动进行数值模拟, 并分别考察进口 压力恒定和进口压力瞬变这两种不同的边界条件所对应的空化过程及流动特征. 模型预测的结 果与文献中已有的实验结果吻合较好. 通过数值分析发现, 超空化的出现引起了流场结构及出 流条件的显著改变, 超空流状态下的液相湍动能分布特征和喷孔出口速度类型有利于柴油射流 的一次雾化过程. 此外, 喷孔上游的压力波动对空化过程有着重大的影响, 无论是部分空化还是 超空化都会随着上游压力的急剧升高或降低而表现出不稳定的特性.

关键词

柴油喷嘴

空化流 双流体模型

压力波动

数值模拟
一些实验研究结果 ${ }^{[1 \sim 5]}$ 表明, 在柴油的高压喷射 过程中喷嘴内部的空化过程及湍流对喷嘴近场的雾化 过程起着重要的作用, 进而影响柴油机的喷雾及燃烧 特性. 为了深入地探讨柴油射流雾化的机理, 对柴油 喷嘴内部的空化流进行全面的研究是很有必要的.

发生于柴油喷嘴中的空化流涉及到湍流、质量传 输、可压缩性和非定常等几乎所有的复杂流动现象. 由 于空化流动本身很复杂以及喷嘴内流场的几何尺度 相当小(量级在 $100 \mu \mathrm{m}$ 左右), 目前的实验技术手段 仍然很难从喷嘴内流中获取比较详实的流场参数. 在此背景下, 数值模拟就成为研究柴油喷嘴内部空 化流动的一种很重要的手段, 进而引起人们的广泛 关注. 至今, 喷嘴空化流的数值模拟方法主要有三类: (i ) 均相流模型方法 ${ }^{[6 \sim 8]}$, 该方法将气液两相混合流 体处理成一均匀介质, 并通过求解气相或液相的体 积分数的输运方程来确定空化区域; (ii) VOF (volume of fluid)方法 ${ }^{[9]}$, 该方法通过引入流体分数体积 函数来计算气液两相的界面, 通常需要大量的网格, 计算量庞大; (iii) 双流体模型方法 ${ }^{[10,11]}$, 该方法分别 建立气液两相的守恒方程, 并通过附加源项的办法 来考虑相间的交互作用, 与均相流模型方法相比, 它 能够反映更多的流场细节, 同时, 所需要的计算量比 VOF方法的少.
基于上面的讨论，本文采用一种双流体模型来 数值模拟一单孔柴油喷嘴内部的空化流动, 分别考 察了进口压力恒定和进口压力瞬变这两种不同的边 界条件所对应的空化过程及流动特征，并将不同流 场结构所对应的喷孔出流条件进行了对比分析. 此 外, 采用文献中有限的实验结果对数学模型和数值 方法的可靠性进行了验证.

\section{1 数学模型描述}

\section{1 双流体模型的基本方程}

本文在计算过程中假定流动是等温的, 则无需 求解能量方程. 依照双流体模型的基本思想, 气液两 相的质量与动量的守衡方程分别如下:

连续性方程:

$$
\frac{\partial\left(\alpha_{k} \rho_{k}\right)}{\partial t}+\nabla \cdot\left(\alpha_{k} \rho_{k} \boldsymbol{V}_{k}\right)=\sum_{l=1, l \neq k}^{2} \Gamma_{k l},
$$

动量方程:

$$
\begin{aligned}
& \frac{\partial\left(\alpha_{k} \rho_{k} \boldsymbol{V}_{k}\right)}{\partial t}+\nabla \cdot\left(\alpha_{k} \rho_{k} \boldsymbol{V}_{k} \boldsymbol{V}_{k}\right)=-\alpha_{k} \nabla p \\
& +\nabla \cdot \alpha_{k}\left(\boldsymbol{\tau}_{k}+\boldsymbol{T}_{k}^{t}\right)+\alpha_{k} \rho_{k} \boldsymbol{g}+\sum_{l=1, l \neq k}^{2} \boldsymbol{M}_{k l}+\boldsymbol{V}_{k} \sum_{l=1, l \neq k}^{2} \boldsymbol{\Gamma}_{k l},
\end{aligned}
$$

(1)及(2)式中, $\Gamma_{k l}$ 和 $\boldsymbol{M}_{k l}$ 分别为相间的质量传输项和动 量传输项, 下面将针对喷嘴空化流中的气液两相来 
建立二者的计算式，其他符号的意义详见参考文 献[10].

\section{2 相间质量传输}

在空化流中, 蒸气相和液相之间的质量传输源 自蒸发过程或凝结过程, 并与气泡的动力行为有关, 则

$$
\Gamma_{12}=\rho_{1} \frac{N}{C_{R}} 4 \pi R^{2} \frac{\partial R}{\partial t}=-\Gamma_{21},
$$

(3)式中, $C_{R}$ 为控制凝结率的经验系数, $C_{R} \geq 1 ; N$ 为气 泡的数密度, 采用文献[12]推荐的公式计算:

$$
N= \begin{cases}N_{0} & \alpha_{1} \leqslant 0.5, \\ 2\left(N_{0}-1\right)\left(1-\alpha_{1}\right)+1 & \alpha_{1}>0.5,\end{cases}
$$

其中, $N_{0}$ 为初始的气核数密度, 本文在计算时取 Fujimoto等人 ${ }^{[13]}$ 推荐的值 $10^{12} . R$ 为气泡的平均半径, 当气泡的数密度和气相的体积分数已知时, $R$ 也唯一 确定, 即

$$
R=\frac{1}{2}\left(\frac{6 \alpha_{1}}{\pi N}\right)^{1 / 3} .
$$

此外, 气泡半径的变化率可由线性化后的Rayleigh方 程推导出 ${ }^{[10]}$, 即

$$
\frac{\partial R}{\partial t}=\frac{\Delta p}{|\Delta p|} \sqrt{\frac{2|\Delta p|}{3 \rho_{2}}} .
$$

上式中，有效压差 $\Delta p$ 的定义为

$$
\Delta p=p_{\mathrm{sat}}-p+\frac{2}{3} C_{E} \rho_{2} k_{2} .
$$

其中, $p_{\text {sat }}$ 为饱和蒸气压力; 右边第三项为考虑湍流的 压力脉动对空化形成的影响而引入的修正项, $C_{E}$ 为 Egler系数, 它取决于当地的湍流水平 ${ }^{[14]}$.

\section{3 相间动量传输}

相间的动量传输通常表示为界面力的形式. 对 于空化流中的气液两相而言, 界面力主要有电力和 湍流扩散力, 则

$$
\boldsymbol{M}_{12}=\boldsymbol{F}_{12}^{\mathrm{D}}+\boldsymbol{F}_{12}^{\mathrm{TD}}=-\boldsymbol{M}_{21},
$$

(8)式中, 电力 $\boldsymbol{F}_{12}^{\mathrm{D}}$ 和湍流扩散力 $\boldsymbol{F}_{12}^{\mathrm{TD}}$ 的计算式分别 如下:

$$
\begin{gathered}
\boldsymbol{F}_{12}^{\mathrm{D}=}-\boldsymbol{F}_{21}^{\mathrm{D}}=C_{\mathrm{D}} \frac{3 \alpha_{1} \rho_{2}}{8 R}\left|V_{2}-\boldsymbol{V}_{1}\right|\left(V_{2}-\boldsymbol{V}_{1}\right), \\
\boldsymbol{F}_{21}^{\mathrm{TD}}=-\boldsymbol{F}_{12}^{\mathrm{TD}}=C_{\mathrm{TD}} \rho_{2} k_{2} \nabla \alpha_{1},
\end{gathered}
$$

其中, $C_{\mathrm{D}}$ 为电力系数, 按照文献[15]所推荐的关系式 计算; $C_{\mathrm{TD}}$ 为湍流扩散系数, 本文计算时取文献 $[16]$ 推
荐的值 0.1 , 该值适用于泡状流的模拟.

气液两相湍流参数的预报基于 $k-\varepsilon$ 模型. 湍动能 方程与湍动能耗散率方程的详细描述以及处理相间 传输项所涉及到的假设条件可参见文献 [10,11], 这 里不再赘述. 此外, 在计算湍流㴝性系数的过程中同 时考虑了剪切引起的和气泡引起的湍流潻性. 气泡 引起的湍流率性采用Sato等人 ${ }^{[17]}$ 提出的公式进行计 算.

\section{2 数值求解}

几何模型的原型为Chaves等人 “实验所采用的 一种单孔柴油喷嘴, 其中, 喷孔具有锐边入口, 其直 径 $d=0.2 \mathrm{~mm}$, 且 $D / d=4, L / d=5, D$ 和 $L$ 分别为喷嘴 上游进口的直径和喷孔长度. 由于几何模型具有轴 对称性, 同时为了减少计算量, 本文进行二维流动的 计算. 计算域及网格结构的示意如图 1. 其中, 对喷 孔处的网格进行局部加密处理, 并通过网格无关性 数值实验来确定各个计算工况所需的网格密度.

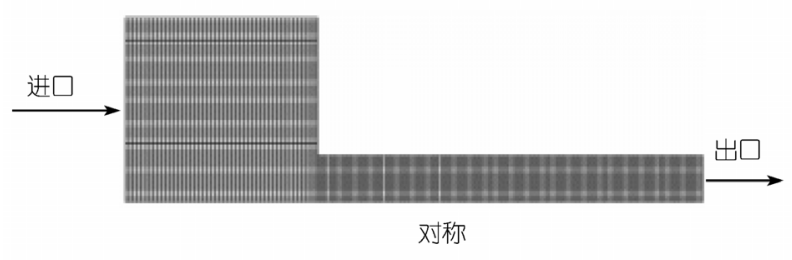

图 1 计算域及网格示意

参照图 1, 数值计算的边界条件为: 上游进口和 喷孔出口均给出压力边界条件; 前后边界和下边界 取对称性边界条件, 即法向速度和各物理量的法向 梯度设为零; 近壁区采用标准的壁面函数法处理, 原 理与单流体的相同.

数值计算在CFD程序—FIRE中进行. 基于有 限体积法, 动量方程中的对流项采用二阶中心差分 和一阶迎风差分相混合的格式进行离散, 混合因子 取为 0.5 ; 连续性方程的对流项采用二阶中心差分格 式离散; 其他方程中的对流项采用一阶迎风差分格 式离散; 方程中的扩散项采用一种特殊的二阶插值 方法 ${ }^{[12]}$ 进行处理; 与时间相关的非定常项采用隐式 格式离散。在空化流场的计算过程中, 采用 SIMPLEC算法将速度场和压力场耦合起来并分步迭 代求解, 进而计算流场其他参数.

\section{3 重要参数定义}

流量系数: 


$$
C_{d}=\frac{q_{m}}{A \sqrt{2 \rho_{2} \Delta P}},
$$

(11)式中, $q_{m}$ 为喷孔中实际的质量流量; $A$ 为喷孔的几 何横截面积; $\Delta P$ 为喷孔进出口压力的差值. $C_{d}$ 被用来 表征喷孔的流动效率.

空化数:

$$
C N=\frac{P_{i}-P_{v}}{P_{i}-P_{b}},
$$

(12)式中, $P_{i}, P_{b}, P_{v}$ 分别为供给压力、喷孔出口背压及 蒸气的压力. 在背压一定的情况下, 空化数 $C N$ 随着 供给压力的增加而减小. 空化数是一个常被用来表 征喷孔内部空化程度的无量纲参数.

\section{4 计算结果与分析}

本文分别对进口压力恒定和进口压力瞬变这两 种不同的边界条件所对应的喷嘴内流进行数值分析. 计算中的工作流体为柴油, 当温度取为 $300 \mathrm{~K}$ 时它的 主要物性参数分别为 ${ }^{[8,18]}$ : 液态密度, $855.6 \mathrm{~kg} / \mathrm{m}^{3}$; 液 态黏度, $2.936 \times 10^{-3} \mathrm{~kg} / \mathrm{ms}$; 蒸气密度, $1.087 \times 10^{-2}$ $\mathrm{kg} / \mathrm{m}^{3}$; 蒸气黏度, $1.81 \times 10^{-6} \mathrm{~kg} / \mathrm{ms}$; 饱和蒸气压力, $120 \mathrm{~Pa}$. 此外, 当经验系数 $C_{R}$ 设为 1, Egler系数 $C_{E}$ 在 1.1 1.2 范围内取值时, 双流体模型预测的结果与实 验结果有较好的一致性.

\section{1 进口压力恒定条件下的流动特性}

在此类工况下，喷嘴进口边界处的压力为定值. 为了在较宽的空化数范围内考察喷嘴内部的流态, 本文设定进口压力的取值范围为 $15 \sim 110 \mathrm{MPa}$. 此外, 出口背压固定为 $6 \mathrm{MPa}$, 它代表着喷油时刻柴油机缸 内的特征压力.

对于定压喷射而言，随着上游进口压力的提高 或者空化数的减小喷孔入口缩脉(vena contracta)处的 速度会越来越高, 其附近的静压进一步降低. 当静压 低于饱和蒸气压力时, 喷孔内流则会从单相流转变 成空化流. 对应于这一流态转变的空化数 $C N$ 被定义 为临界空化数 $C N_{\text {crit }}$. 针对 $L=1 \mathrm{~mm}, d=0.2 \mathrm{~mm}$ 的喷 孔, Chaves等人 ${ }^{[1]}$ 研究了其内部发生空化流的临界条 件, 结果如图 2 所示. 可以看出, 出口背压为 $6 \mathrm{MPa}$ 时, 大致需要 $30 \mathrm{MPa}$ 左右的压差才能使喷孔内部产 生空化流, 则相应的临界空化数 $C N_{\text {crit }}$ 约为 1.2 . 值得 一提的是, 其他出口背压条件对应的 $C N_{\text {crit }}$ 也在 1.2 左 右，这可能是上述喷孔所具有的一个特征.

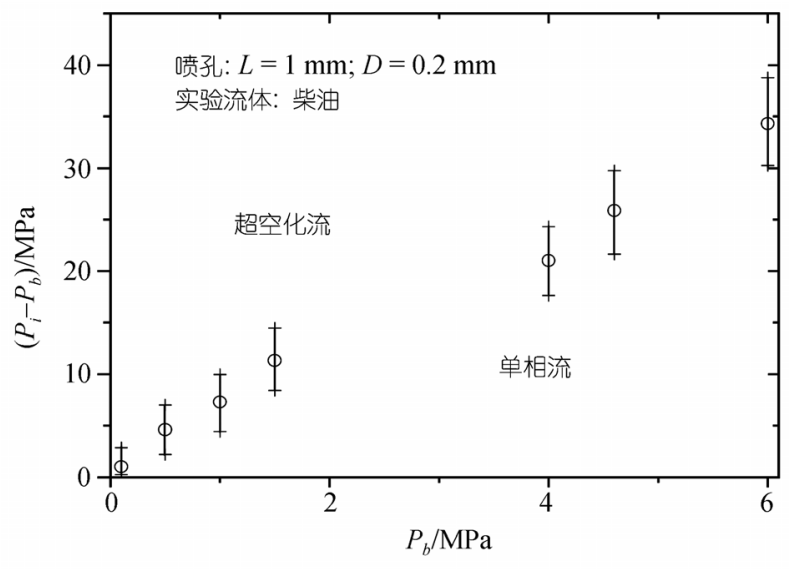

图 2 喷孔内产生空化流的压力条件

喷孔的流量系数是影响柴油喷雾特性的重要参 数之一. 随着空化的出现, 喷孔的有效流通面积会发 生变化, 这必将导致流量系数的改变. 前人通过实验 手段和一维流动的理论分析方法来考察流量系数, 主要结论如下: 在单相流状态下 $\left(C N>C N_{\text {crit }}\right)$, 流量 系数的变化主要依赖于雷诺数 $R e^{[19]}$, 并通常接近于 一常值; 在空化流状态下 $\left(C N<C N_{\text {crit }}\right)$, Nurick ${ }^{[20]}$ 的研 究显示, 流量系数的变化主要取决于空化数, 并提出 如下的关系式:

$$
C_{d}=C_{c} \sqrt{C N},
$$

其中, $C_{c}$ 为喷孔的收缩系数. Payri等人 ${ }^{[4]}$ 的实验研究 也总结出上面的关系, 只是临界空化数 $C N_{\text {crit }}$ 和收缩 系数 $C_{c}$ 因实验条件的差异而取值不同. 参照(13)式, 本文所用喷孔的收缩系数 $C_{c}$ 可以处理成 $C N$ 逼近于 1 时所对应的最小流量系数, 也由Chaves等人的实验 结果得出, 约为 0.705 .

图 3 所示为该喷孔的流量系数随空化数的变化 情况. 其中, 将双流体模型预测的结果与 Chaves 等 人的实验结果以及 Nurick 关系式的计算结果进行了 比较. 可以看出, 三者有着很好的一致性. 双流体模 型所预测的临界空化数也较为合理(接近于 1.2), 同 时数值计算的结果进一步地验证了在空化流状态下 流量系数与空化数的平方根近似成线性关系, 这说 明本文采用的数学模型和数值方法是可靠的.

下面以进口压力取 $70 \mathrm{MPa}$ 为例，对喷孔内流的 流态以及相应的流场参数的变化情况进行考察. 图 4 所示为气相体积分数在喷孔内部的分布随时间的变 化情况. 可以看出, 喷嘴内流在很短的时间内依次历 经单相流、空化初生和部分空化流, 并最后稳定在超 


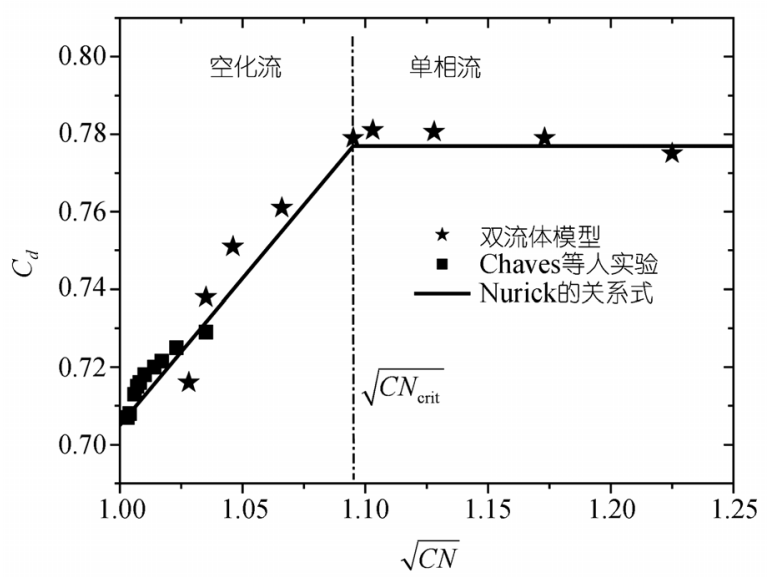

图 3 喷孔流量系数随空化数的变化

空化流. 喷孔内部可能出现的流态基本上可划分为 上述四种. 但与前三种流态不同的是, 超空化流一般 只出现在高压喷射的过程中, 这可由图 2 的结果得知. 在前三种流态下, 喷孔出口附近的流场中不存在空 化区, 出流仅为液相, 则液柱直径等于喷孔直径. 而 在超空化流状态下, 空化区沿着喷孔内壁呈薄膜状 一直蔓延至喷孔出口, 出流由液相变为气液两相, 并 只在喷孔的轴心区域存在着完整的液柱, 其有效直 径明显小于喷孔直径. 显然, 在超空化流形成之后喷 雾的初始条件发生了很大的改变, 这将直接影响到 液柱在喷嘴近场中的破碎程度, 因而需要对这种变 化予以详细分析.

图 5 所示为无因次液相湍动能在喷孔内部的分 布随空化形态的变化情况. 其中, 无因次液相湍动能 定义为液相湍动能与同一计算时刻的流场中最大的 液相湍动能之比. 可以看出, 随着喷孔内部流态的改 变, 液相湍动能在喷孔内部的分布形态是不断发生 变化的. 总体上, 大的液相湍动能出现的位置与空化 区出现的位置基本一致, 均在壁面附近. 随着空化的 产生及空化区域向下游的延伸, 流场中大的液相湍 动能所集中的区域也随之向下游扩展, 这说明喷孔 入口附近的流动分离现象所诱发的旋浴结构以及气 液两相之间的交互作用有利于提高当地流场的湍流 度. 值得注意的是, 在超空化流状态下液相湍动能在 出口附近流场中的分布是极不均匀的. 其中, 大的液 相湍动能集中于液柱的表面区域, 而小的液相湍动 能则主要分布在液柱的中心区域, 并且二者在量级 上有着很大的差异, 以致于两区的分界十分明显. 液 相湍动能的这种分布格局将使得液柱的表面附近存
在着很大的径向脉动速度及相应的附加应力, 进而 加强了液柱表面的初始扰动水平, 这对液柱离开喷 孔后的第一阶段破碎是有利的.

$$
t=5.6 \mu \mathrm{s} \text { 单相流 }
$$

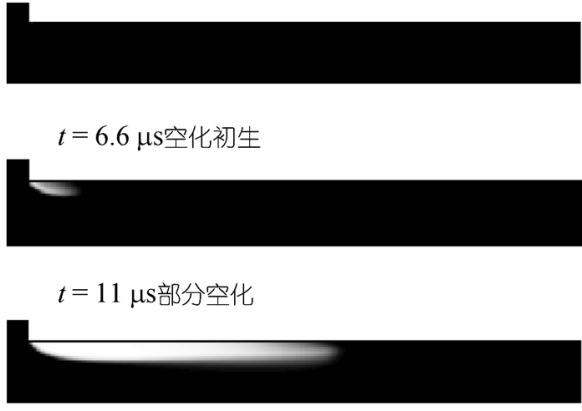

$t=15 \mu \mathrm{s}$ 超空化

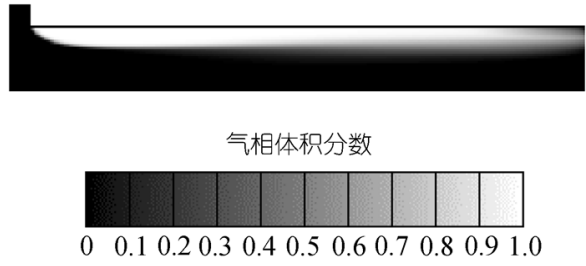

图 4 喷孔内分气相体积分数布的演变 $\left(P_{\mathrm{i}}=70 \mathrm{MPa}\right)$

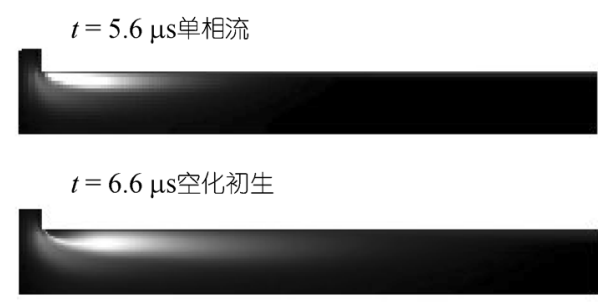

$$
t=11 \mu \mathrm{s} \text { 部分空化 }
$$

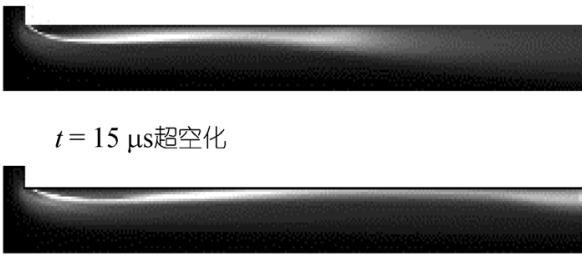

无因次液相湍动能

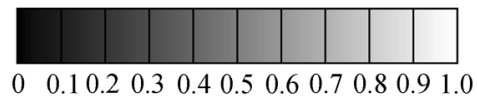

图 5 喷孔内无因次液相湍动能布的演变 $\left(\boldsymbol{P}_{\mathrm{i}}=70 \mathrm{MPa}\right)$

图 6 所示为进口压力取 $70 \mathrm{MPa}$ 时喷孔出口处轴 向速度剖面随空化发展的变化情况. 其中, 以出流方 向的轴向速度为正. 从图 6 可以看出, 在超空化流形 成之前, 轴向速度从边界层向主流区的过渡是比较 
平缓的. 但是, 主流区的轴向速度会随着空化的发生 以及空化区向下游的发展而呈增加的趋势, 这是由 于在越来越长的喷孔流道内有效流通面积的减小导 致了流场速度分布的重组，并使速度向主流区域集 中. 当超空化流形成时，整个喷孔流道内的有效流通 面积均减小, 位置与主流区对应的液柱将具备更大 的轴向速度. 由上面的分析可知, 在超空化流状态下 液柱的有效直径虽然减小，但它上面各点的轴向速 度均有大幅度的提高，当液柱与喷嘴近场的气相介 质接触时气液两相间的相对速度也会增加，这将使 气动力的作用得到加强, 进而有利于雾化.

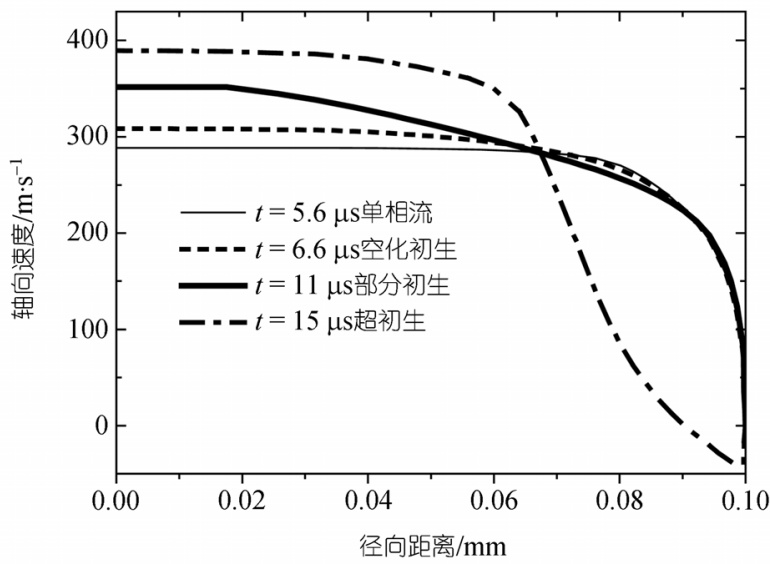

图 6 喷孔出口轴向速度剖面的演变 $\left(P_{\mathrm{i}}=70 \mathrm{MPa}\right)$

综上，超空化流的出现是空化开始直接作用于 喷射液柱以及喷雾初始条件发生重大变化的一个标 志. 这说明超空化流发生前后所对应的初次雾化过 程是不一样的, 对它们发生的机理要加以区别. 此外, 在目前柴油机的高压喷射过程中, 超空化流是一种 常见的现象，则需要综合考虑流量系数的变化、湍动 能与轴向速度的分布特征对初次雾化的影响. 如果 忽略这些因素的影响，对柴油高压喷雾形成机理的 解释将是不完备的.

\section{2 进口压力瞬变条件下的流动特性}

到目前为止, 大部分的实验研究和数值研究都 是在考察定压边界条件下的喷孔空化流特性. 但是 在实际的柴油高压喷射过程中，喷孔上游的流场往 往是瞬变的, 这主要是由针阀沿径向和轴向的不稳 定运动所造成的. Chaves 等人 ${ }^{[21]}$ 对针阀腔内(靠近喷 孔入口)的流场压力进行了测量，其结果显示，该区 域内的流场压力是剧烈波动的, 波动频率的量级甚
至可达到 25 55 kHz. 为了考察上游的高频压力波动 对喷孔内部空化流特性的影响, 本文将喷嘴进口边 界处的压力设置成周期性变动的形式，如图 7 所示. 其中, 波动幅度和波动频率的量级参照了 Chaves等 人 ${ }^{[21]}$ 的实验结果. 此外, 出口背压仍然固定为 $6 \mathrm{MPa}$.

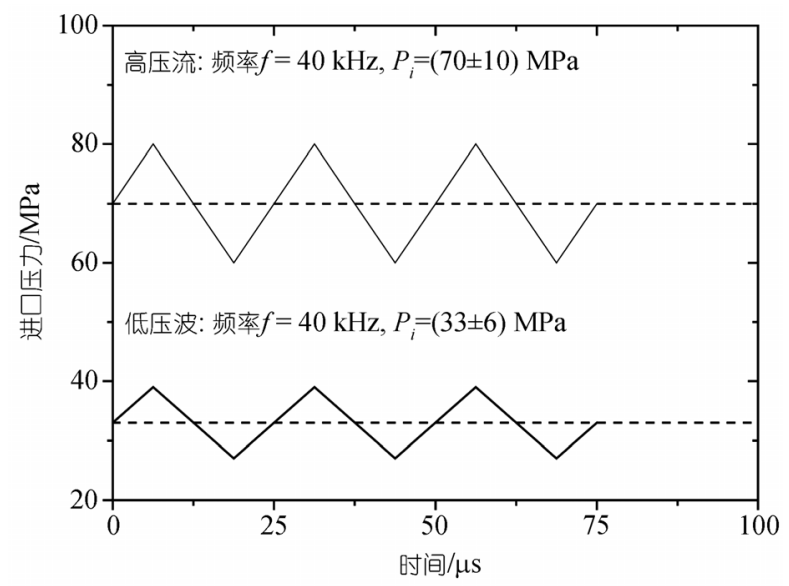

图 7 喷嘴进口压力随时间的变动

图 8 所示为两种类型的进口压力波动所对应的 喷孔内部空化形态的演变情况. 可以看出, 在进口压 力的一个波动周期内, 无论是低压波动对应的部分 空化过程还是高压波动对应的超空化过程均表现得 很不稳定. 理论上讲, 进口压力的急剧升高引起了空 泡的溃灭, 进而使得空化区域缩短或变薄; 进口压力 的瞬时下降则会促进空泡的成长，进而使得空化区 域增加或变厚. 但值得注意的是, 图 8 中最强的空化 不是出现在波谷对应的时刻，而最弱的空化也并非 出现在波峰对应的时刻, 空化过程明显滞后于进口 压力的变动. 例如, 图 8(b)中进口压力的峰值出现在 $t=31.25 \mu \mathrm{s}$, 但最弱的空化出现 $t=34 \mu \mathrm{s}$ 左右. 造成 这种现象的原因可能是: 非空化区的液体是几近不 可压的, 这样进口压力的变动将以趋于无限的速度 传播到整个液相流场，但速度场的相应变化则会由 于动量输运方程中各项的制约而出现滞后, 从而延 缓了流场其他参数以及空化区域的变化.

此外, 结合针对于图 4 6 的分析可知, 低压波动 引起的不稳定部分空化过程虽然造成了喷孔内部空 化程度的反复, 但并不会较大程度地改变喷孔出流 的条件, 这是因为出流仍然为只有液相的流动, 如图 8(a)所示. 但在高压波动的情况下，喷孔出口附近的 区域会随着进口压力的变动而呈现出截然不同的流 


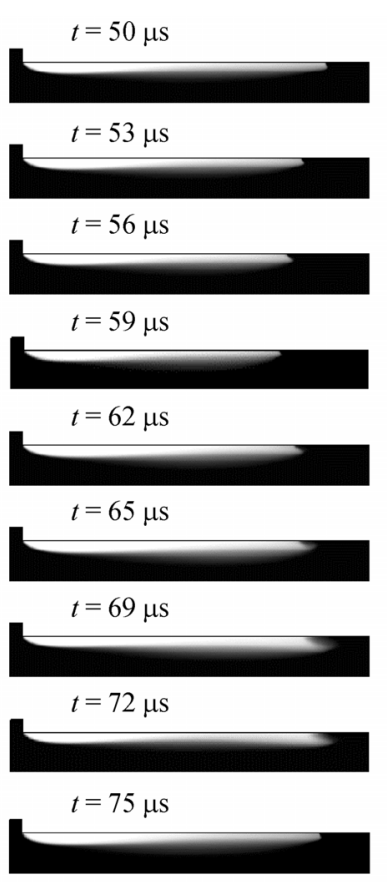

\section{气相体积分数}

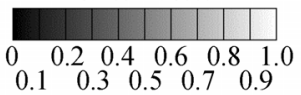

(a)

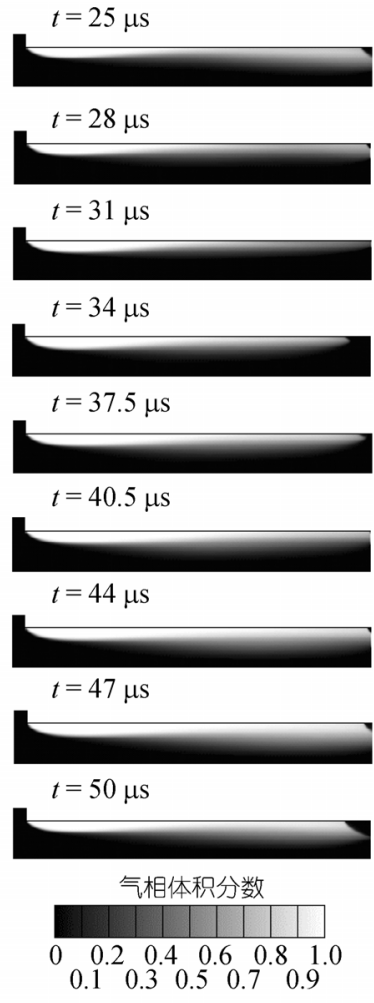

(b)

图 8 进口压力波动引起的喷孔内不稳定的空化过程 (a) 低压波; (b) 高压波

态, 这与进口压力恒定以及上述低压波动对应的情 况有着很大的差异. 如图 8(b)所示, 当计算时刻在 34

$\mu \mathrm{s}$ 左右时, 超空化甚至会退化成部分空化. 很显然, 不稳定的超空化行为所对应的喷孔出流条件也是很 不稳定的, 时而为单相流, 时而为两相流, 并极为复 杂. 在柴油机的喷射过程中, 压力波动是真实存在的, 因此在确定喷雾初始条件时需充分考虑压力波动所 诱发的喷孔内流特性.

\section{5 结论}

本工作利用双流体模型对柴油喷孔空化流在进 口压力恒定和瞬变两种不同模式下的特性进行数值 分析, 主要结论如下:

（1）基于双流体模型的空化流模拟得到了与相 应条件下的实验结果相当吻合的结果. 数值计算预 测的临界空化数较为合理, 并进一步地验证了 Nurick 的关系式, 这说明本文采用的模型和数值方 法能够较好地预报喷嘴空化流的主要特性.

(2) 超空化流的出现是空化开始直接作用于喷 射液柱及喷雾初始条件发生重大变化的一个标志. 在超空化流状态下, 喷射液柱所具备的轴向速度及 湍动能的分布特征有利于它离开喷孔后的第一阶段 破碎.

(3) 喷孔内部的空化过程对边界条件比较敏感, 部分空化和超空化都会因进口压力的波动而变得不 稳定. 不稳定的超空化使得不同时刻的喷孔出流条 件有着明显的差异, 需要引起重视.

\section{参考文献}

1 Chaves H, Knapp M, Kubitzek A. Experimental study of cavitation in the nozzle hole of diesel injectors using transparent nozzles. SAE Paper 950290, 1995

2 Badock C, Wirth R, Fath A, et al. Investigation of cavitation in real size diesel injection nozzles. Int J Heat Fluid Flow, 1999, 20(5): 538 - 544[DOI]

3 Soteriou C, Andrews R, Smith M. Further studies of cavitation and atomization in diesel injection. SAE Paper 1999-01-1486, 1999

4 Payri F, Bermudez V, Payri R, et al. The influence of cavitation on the internal flow and the spray characteristics in diesel injection nozzles. Fuel, 2004, 83(4): 419-431[DOI]

5 Sou A, Hosokawa S, Tomiyama A. Effects of cavitation in a nozzle on liquid jet atomization. Int J Heat Mass Transfer, 2007, 50(17-18): 3575-3582[DOI]

6 Yuan W, Schnerr G H. Numerical simulation of two-phase flow in injection nozzles: interaction of cavitation and external jet formation. J Fluids Eng, 2003, 125(6): 963-969 $\underline{\text { [DOI] }}$

7 Kubo M, Araki T, Kimura S. Internal flow analysis of nozzles for DI diesel engines using a cavitation model. JSAE Rev, 2003, 24(3): $255-261[$ DOI]

8 Jia M, Hou D, Li J, et al. A micro-variable circular orifice fuel injector for HCCI-conventional engine combustion-part I numerical simulation of cavitation. SAE Paper 2007-01-0249, 2007

9 Marcer R, LeGouez J M. Simulation of unsteady cavitating flows in diesel injector with an improved VOF-method. In: Proc of 17th ILASS-Eu Conf, September 2-6, 2001, Zurich Switerland 